\title{
ST Reha: changement de direction dans le développement du tarif
}

\section{Beatrix Meyer}

Cheffe de la division Médecine et tarifs hospitaliers, FMH

\author{
Le conseil d'administration de SwissDRG SA a décidé de repousser l'introduction de \\ ST Reha pour que la structure tarifaire puisse être développée sur la base des codes \\ de la classification des interventions chirurgicales (CHOP) et introduite le $1^{\text {er }}$ janvier \\ 2022. Conjointement avec les sociétés de discipline, la FMH a déjà élaboré des pro- \\ positions sur la manière de prendre en compte les prestations de réadaptation dans \\ la CHOP.
}

\section{Réadaptation neurologique, gériatrique, pulmonaire, cardiaque, musculo-squelettique, interniste/oncologique, psychosomatique et pédiatrique.}

\begin{abstract}
C'est définitif, le conseil d'administration de SwissDRG

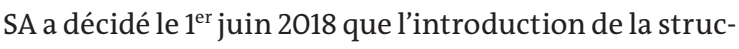
ture tarifaire ST Reha version 0.5 n'aurait pas lieu alors que celle-ci était finalisée. La FMH regrette cette décision après les longues années de travaux investies dans son développement. Les assureurs-maladie et les cantons avaient critiqué le modèle de lien indirect avec les prestations. C'est pourquoi SwissDRG SA entend développer une nouvelle structure tarifaire avec un modèle de lien direct. Il s'agit pour cela de saisir les prestations fournies par les cliniques de réadaptation selon les codes de la CHOP et de les intégrer dans la structure
\end{abstract}

Figure 1: prestation de base de la réadaptation

(sur la base des propositions de la FMH et des sociétés de discipline)

1. Caractéristiques minimales communes à tous les types de réadaptation: Bulletin d'hospitalisation

Plan de traitement

Indications sur la thérapie et le coaching, par ex. durée d'une unité de traitement

Visite du médecin-chef

Coordination et discussion en équipe du cas de réadaptation

Planification de sortie

2. Caractéristiques minimales spécifiques à chaque type de réadaptation:

Paramètres spécifiques complémentaires au bulletin d'hospitalisation

Visites supplémentaires, hormis celle du médecin spécialiste

- Définition de la thérapie et du coaching, par ex. quelle thérapie doit être obligatoirement fournie et dans quelle mesure; quelles formes différentes de thérapies peuvent être combinées pour le patient; comment est définie la densité minimale des traitements par semaine

Planification de sortie supplémentaire

Evaluation de sortie, en plus de l'établissement du score ADL (Activities of Daily Living)

Vous trouverez une description détaillée (en allemand) des caractéristiques minimales des prestations de base en réadaptation sur notre site www.fmh.ch $\rightarrow$ Tarifs hospitaliers $\rightarrow$ Réadaptation ST Reha. tarifaire ST Reha. Par ailleurs, la prochaine structure tarifaire comportera un système d'arborescence décisionnelle. L'introduction de ce nouveau modèle tarifaire est prévue pour le $1^{\mathrm{er}}$ janvier 2022.

\section{Changement de direction prévisible}

Pour la FMH, ce changement de direction n'est pas une surprise. Le conseil d'administration de SwissDRG SA avait en effet déjà envisagé en 2016 de procéder à l'intégration des codes CHOP pour passer, à moyen terme, à une structure tarifaire axée davantage sur les prestations. La même année, la réadaptation n'étant pas suffisamment bien prise en compte dans la CHOP, la FMH a commencé ses travaux en vue de définir les prestations relevant de ce domaine, avec l'objectif de trouver une solution permettant de ne créer qu'un nombre limité et absolument nécessaire de nouveaux codes CHOP et de maintenir ainsi les charges administratives pour saisir ces prestations au plus bas.

\section{La FMH et les sociétés de discipline définissent les prestations de base}

Pour le corps médical, il est primordial de garantir la qualité de la réadaptation et, par conséquent, de définir les prestations minimales pour les patients admis en réadaptation hospitalière. Conjointement avec les sociétés de discipline, la FMH a élaboré des propositions visant à définir les prestations de base de chaque type de réadaptation ${ }^{1}$ en partant de plusieurs caractéristiques minimales. Ces dernières sont réparties en deux catégories. D’un côté, les caractéristiques communes à 
2 La FMH avait préconisé une solution sans codage CHOP des prestations de base. Une solution possible aurait été de les cartographier par le biais d'une redistribution des principaux centres de coûts dans la statistique médicale des hôpitaux. L'OFS a préféré ne pas suivre cette proposition de la FMH. tous les types de réadaptation (par ex. un plan de traitement doit toujours être fixé trois jours au plus tard après l'hospitalisation); de l'autre, les caractéristiques spécifiques prévues en fonction du type de réadaptation (cf. fig. 1). A cela s'ajoute la définition des qualifications et des exigences minimales (qui doivent être remplies respectivement présentes) pour fournir une prestation. Il s'agit notamment de clarifier quelle est la qualification requise pour diriger l'équipe de traitement ou quels thérapeutes doivent la composer.

\section{Prestations supplémentaires pour la réadaptation}

En plus de ces prestations de base, les cliniques de réadaptation peuvent aussi être amenées à fournir différentes prestations supplémentaires en fonction de leur spécialisation et du collectif de leurs patients. Il peut s'agir par exemple de prestations de soins liées à l'indication clinique comme une surveillance étroite des paramètres vitaux après un traumatisme crânien ou le traitement complexe d'une plaie. Conjointement avec les sociétés de discipline, la FMH a déjà travaillé afin de définir ces prestations supplémentaires. En 2017, les sociétés de discipline ont déposé leurs propositions pour les codes CHOP à l'Office fédéral de la statistique (OFS). Avec le soutien de la
Correspondance:

FMH

Baslerstrasse 47

CH-4600 Olten

Tél. 0313591111

Fax 0313591112

tarife.spital[at]fmh.ch
FMH, elles ont par ailleurs demandé à ce que quatre des codes CHOP existants soient adaptés en fonction de la réadaptation (cf. les propositions mises en œuvre, fig. 2).

\section{Ces définitions sont intégrées dans la CHOP 2019}

L'OFS et son groupe d'experts dans lequel siègent des représentants de SwissDRG SA, des assureurs, des cantons, de $\mathrm{H}+$ et de la FMH ont examiné et discuté plusieurs fois les définitions des prestations de base et des prestations supplémentaires élaborées par la FMH et ses sociétés de discipline. Les suggestions des partenaires ont été entendues et intégrées lorsque c'était possible. Entre-temps, l'OFS a repris ces définitions dans la prochaine version de la CHOP qui entrera en

La nouvelle version ST Reha repose sur la représentativité des prestations de base et des prestations supplémentaires dans la CHOP.

vigueur le $1^{\text {er }}$ janvier 2019. Il est important que les cliniques de réadaptation saisissent bien les codes $\mathrm{CHOP}$ pour que SwissDRG SA puisse calculer son nouveau modèle de ST Reha sur la base de données fiables. Pour la prestation de base, il suffit de saisir un seul code CHOP par cas de réadaptation hospitalière ${ }^{2}$, auquel s'ajoute le code des éventuelles prestations supplémentaires. Il est primordial de déterminer proprement les prestations car cela permet d'identifier rapidement la nécessité ou le besoin d'adapter ou de compléter les prochaines versions de la CHOP. Les propositions dans ce sens peuvent être déposées auprès de l'OFS dans le cadre d'une procédure annuelle.

\section{Poursuivre les travaux rapidement}

Les définitions des prestations de base et des prestations supplémentaires pour la réadaptation posent un jalon important en vue du développement de la nouvelle structure tarifaire ST Reha incluant les codes CHOP. Dans une prochaine étape, il s'agira pour SwissDRG SA et les partenaires d'établir un concept en vue de cadrer ce développement mais aussi d'élaborer des solutions pour la rémunération des prestations onéreuses tout en tenant compte des prestations qui ne sont pas spécifiques à la réadaptation, comme les dialyses ou les médicaments chers. Il est aussi nécessaire d'avoir une délimitation claire entre ST Reha et les autres structures tarifaires. Il reste donc encore passablement à faire d'ici l'introduction de ST Reha au $1^{\text {er }}$ janvier 2022. 